\title{
A CONNECTIVITY LEMMA FOR THE ALBANESE MAP
}

\author{
M. Rovinsky
}

\begin{abstract}
I show that for any complex projective variety $X$ and a sufficiently large integer $N$ all the fibers of Albanese map of the $N$-th symmetric power of $X$ are dominated by connected projective varieties with vanishing $H^{1}$. This result reduces certain problems on 0 -cycles on smooth varieties to those on smooth surfaces with vanishing $H^{1}$ 's.
\end{abstract}

Let $X$ be a smooth projective complex variety. After a choice of a point on $X$ one defines the Albanese map from $X$ to an abelian variety $\operatorname{Alb} X$ (called the Albanese variety of $X$ ) by the following universality property: any morphism from $X$ to an abelian variety $B$, which sends the marked point to the identity element of $B$ factors uniquely through a homomorphism $\operatorname{Alb} X \longrightarrow B$.

It is well-known, that in general the fibers of the Albanese map are not connected (e.g., for a general hyperplane section of a projective embedding of the product of a two-dimensional abelian variety and the projective line). However, Lemma 1.2 below shows that the fibers are connected for "sufficiently averaged" Albanese map.

Moreover, let $F$ be an algebraically closed field of characteristic zero and $X$ be a smooth connected projective variety over $F$. Then one has the following

Proposition 0.1. If an adequate equivalence relation on cycles (see [Sa]) identifies each pair of points on each smooth connected projective surface over $F$ with trivial Albanese variety, then the group of equivalence classes of 0-cycles of degree zero on $X$ is a quotient of the group of F-points of $\operatorname{Alb} X$.

Remark. A particular case of a well-known conjecture of Beilinson and Bloch predicts that the Albanese map from the Chow group of degree-zero 0-cycles on a smooth projective variety over $\overline{\mathbb{Q}}$ is bijective. Here we get the following reduction of this.

Corollary 1. If for a field $F$ as above one has $C_{0}(Y)^{0}=0$ for all smooth connected projective surfaces $Y$ over $F$ with $\operatorname{Alb} Y=0$, then the Albanese map $C H_{0}(X)^{0} \longrightarrow(\operatorname{Alb} X)(F)$ is bijective for any smooth projective variety $X$ over $F$.

Received September 5, 2000.

The author was partially supported by the Paul and Gabriella Rosenbaum fellowship while at MIT. 
The Proof is divided into three lemmas, where we assume that $X$ is a complex manifold and keep the following notations: $h=\operatorname{dim} \operatorname{Alb} X, p: X \longrightarrow \operatorname{Alb} X$ is an Albanese map, and $p_{N}: X^{N} \longrightarrow \operatorname{Alb} X,\left(x_{1}, \ldots, x_{N}\right) \mapsto p\left(x_{1}\right)+\ldots+p\left(x_{N}\right)$.

Lemma 1.1. $p_{M}$ are open for all $M \geq h^{2}+h$.

Proof. Let $M \geq h^{2}+h$ be an integer, and let $r=[M / h], M_{1}=\cdots=M_{r-1}=h$ and $M_{r}=h+h\{M / h\}$. We have to show that for any point $x=\left(x_{1}, \ldots, x_{r}\right) \in$ $X^{M_{1}} \times \cdots \times X^{M_{r}}=X^{M}$ and any neighborhood $U$ of $x$ the image $p_{M}(U)$ contains a neighborhood of the image $p_{M}(x)$. Shrinking $U$, if necessary, we may assume that $U=U_{1} \times \cdots \times U_{r} \subseteq X^{M_{1}} \times \cdots \times X^{M_{r}}=X^{M}$ for some open subsets $U_{j} \subseteq X^{M_{j}}$.

For each $1 \leq j \leq r$ there is a divisor $D_{j} \subset \operatorname{Alb} X$ such that $p_{M_{j}}$ is open (of maximal rank) over the complements to $D_{j}$, and therefore, $p_{M}$ is open (of maximal rank) over the complement to the pull-back of any $D_{j}$ under the projection of $X^{M}$ onto $X^{M_{j}}$. Then for each $1 \leq j \leq r$ the image of $\prod_{i=1}^{j-1} U_{i} \times$ $\left(U_{j}-p_{M_{j}}^{-1}\left(D_{j}\right)\right) \times \prod_{i=j+1}^{r} U_{i}$ is open. It remains to check that the union $V$ of such open subsets in $\operatorname{Alb} X$ contains $p_{M}(x)$.

There is such a collection of smooth curves $x_{1} \in C_{1} \subseteq X^{M_{1}}, \ldots, x_{r} \in$ $C_{r} \subseteq X^{M_{r}}$ that the restriction $\bar{p}_{M}$ of $p_{M}$ to $C_{1} \times \cdots \times C_{r}$ is surjective and $C_{j} \cap U_{j} \cap p_{M_{j}}^{-1}\left(D_{j}\right) \subseteq\left\{x_{j}\right\}$. Clearly,

$$
\begin{aligned}
\left(\bigcup_{j=1}^{r} \prod_{i=1}^{j-1} U_{i} \times\left(U_{j}-p_{M_{j}}^{-1}\left(D_{j}\right)\right) \times \prod_{i=j+1}^{r} U_{i}\right) \bigcap\left(C_{1} \times \cdots \times C_{r}\right)= & \\
& \prod_{j=1}^{r}\left(C_{j} \bigcap U_{j}\right)-\{x\} .
\end{aligned}
$$

As the irreducible components of fibers of $\bar{p}_{M}: C_{1} \times \cdots \times C_{r} \longrightarrow \operatorname{Alb} X$ are of positive dimension, there is a curve $C$ on $C_{1} \times \cdots \times C_{r}$ passing through $x$ and projecting to $p_{M}(x)$, and thus, $V$ contains $p_{M}(x)$.

Lemma 1.2. For $N$ sufficiently large all fibers of $p_{N}$ are connected.

Remark. It is shown in $[\mathrm{M}]$ that the fibers of $p_{N}$ are irreducible modulo $\mathfrak{S}_{N^{-}}$ action.

The Proof is an elaboration of the following simple remark. Suppose that a morphism to a connected target is surjective on each connected component of the source. Then the number of connected components of the source does not exceed the number of connected components of any fiber. We will apply this remark to $\varrho_{a}$.

Obviously, $p_{M}$ is surjective for large $M$, so, by Lemma 1.1, we may assume that $p_{M}$ is open in the complex topology, but has non-connected fibers. Fix some $k$ with surjective $p_{k}$. 
We denote by $\varrho_{a}$ the restriction of the projection $\mathrm{pr}_{2}: X^{M+k} \longrightarrow X^{k}$ to the fiber

$$
p_{M+k}^{-1}(a)=\left\{\left(x_{1}, \ldots, x_{M+k}\right) \in X^{M+k} \mid p_{M}\left(x_{1}, \ldots, x_{M}\right)=a-p_{k}\left(x_{M+1}, \ldots, x_{M+k}\right)\right\}
$$

of $p_{M+k}$ over a point $a \in \operatorname{Alb} X$. Then $\varrho_{a}$ is also surjective on each connected component of $p_{M+k}^{-1}(a)$ for the following reason. For a point $\left(x_{1}, \ldots, x_{M+k}\right)$ on a given connected component of $p_{M+k}^{-1}(a)$ fix its neighborhood in the complex topology of type $U_{1} \times U_{2}$ in $X^{M} \times X^{k}$ intersecting no other connected components of $p_{M+k}^{-1}(a)$. Then $p_{M}\left(U_{1}\right)$ contains a neighborhood $V$ of the point $p_{M}\left(\left(x_{1}, \ldots, x_{M}\right)\right)$ in $\operatorname{Alb} X$, so $\varrho_{a}\left(U_{1} \times U_{2} \cap p_{M+k}^{-1}(a)\right)$ contains a non-empty open subset $U_{2} \cap p_{k}^{-1}(a-V) \ni\left(x_{M+1}, \ldots, x_{M+k}\right)$ in $X^{k}$.

One can organize all these varieties in the Cartesian square

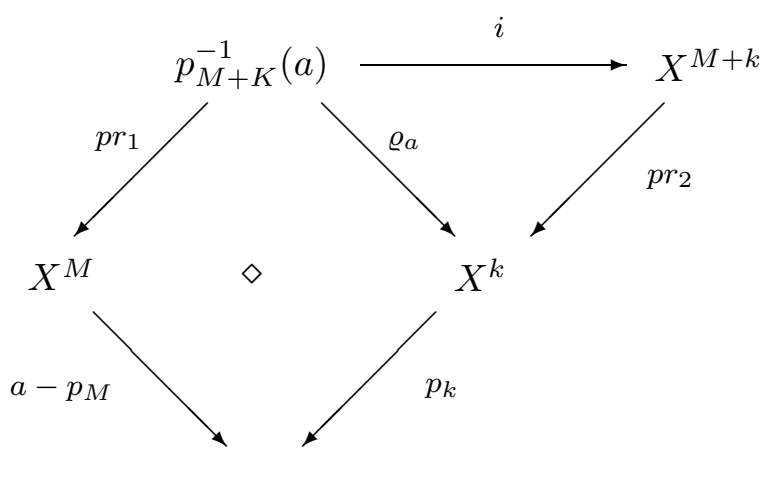

AlbX

As any étale morphism from a proper variety to $\operatorname{Alb} X$ induces an embedding of fundamental groups, but $p_{M}: X^{M} \longrightarrow \operatorname{Alb} X$ induces a non-injective surjection of the fundamental groups, the proper variety $\operatorname{Spec}\left(p_{M *}\left(\mathcal{O}_{X^{M}}\right)\right)$ from the Stein decomposition of $p_{M}$ cannot be étale over $\operatorname{Alb} X$, since $\operatorname{Spec}\left(p_{M *}\left(\mathcal{O}_{X^{M}}\right)\right) \neq$ $\operatorname{Alb} X$. This means that there is a point $\alpha$ of $\operatorname{Alb} X$ with a strictly less quantity of connected components of the fiber of $p_{M}$ over $\alpha$ than that of a general fiber of $p_{M}$.

As each fiber of $\varrho_{a}$ is isomorphic to a fiber of $\left(a-p_{M}\right)$, we can find a point $q$ of $X^{k}$ with a strictly less quantity of connected components of the fiber of $\varrho_{a}$ over $q$ than that of a general fiber of $p_{M}$.

This implies that the number of connected components of $p_{M+k}^{-1}(a)$ is strictly less than the number of connected components of a general fiber of $p_{M}$. So we can find such $N_{0}$ that all the fibers of $p_{N_{0}}$ are connected. For any $N \geq 2 N_{0}$ all the fibers of $p_{N}$ are connected.

For any positive integer $N$ there is a simply-connected projective surface $Y_{N}$ with a free action of the symmetric group $\mathfrak{S}_{N}$, constructed in [Se] as a complete intersection. 
Lemma 1.3. Let $N$ be sufficiently large, and let $\hat{p}_{N}:\left(X^{N} \times Y_{N}\right) / \mathfrak{S}_{N} \longrightarrow \operatorname{Alb} X$ be given by $\left(x_{1}, \ldots, x_{N} ; y\right) \mapsto p\left(x_{1}\right)+\ldots+p\left(x_{N}\right)$.

Then for any point a of $\operatorname{Alb} X$ one has $H^{1}\left(\hat{p}_{N}^{-1}(a)\right)=0$.

Proof. Assume that $k$ is large enough, so that the fibers of $p_{k}$ are connected, and consider the following two Leray spectral sequences

$$
\begin{gathered}
E_{2}^{s, t}(k, M)=H^{s}\left(X^{M}, R^{t} \operatorname{pr}_{1 *}(\mathbb{Q})\right) \Rightarrow H^{s+t}\left(p_{M+k}^{-1}(a) ; \mathbb{Q}\right), \\
E_{2}^{s, t}(k)=H^{s}\left(\operatorname{Alb} X, R^{t} p_{k *}(\mathbb{Q})\right) \Rightarrow H^{s+t}\left(X^{k} ; \mathbb{Q}\right)
\end{gathered}
$$

The Cartesian square (1) gives $R^{q} \operatorname{pr}_{1 *}(\mathbb{Q})=\left(a-p_{M}\right)^{*}\left(R^{q} p_{k *}(\mathbb{Q})\right)$. Clearly, the pull-back $\left(a-p_{M}\right)^{*}: E_{2}^{s, t}(k) \longrightarrow E_{2}^{s, t}(k, M)$ induces an isomorphism on $E_{2}^{0, t}$ and commutes with differentials.

Since $H^{*}(\operatorname{Alb} X ; \mathbb{Q})=\wedge^{*} H^{1}(X ; \mathbb{Q})$ and due to the Künneth formula, for $k \geq \operatorname{dim} \operatorname{Alb} X$ there is a commutative diagram

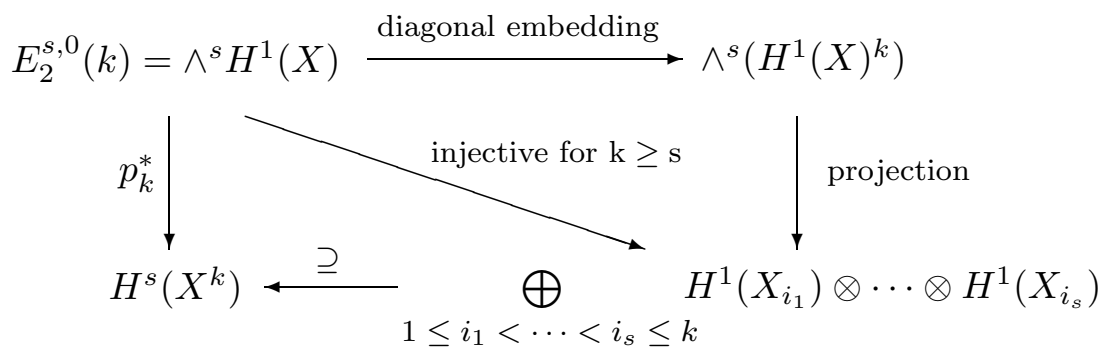

where $X_{i}$ is the $i$ th copy of $X$ in $X^{k}$, and thus, the pull-back $p_{k}^{*}: E_{2}^{s, 0}(k) \longrightarrow$ $H^{s}\left(X^{k}\right)$ is injective for $s \leq k$. This means that $E_{2}^{s, 0}(k)$ coincides with $E_{\infty}^{s, 0}(k)$ and, in particular, that $d_{2}^{s-2,1}(k)=0$.

From the commutative diagram

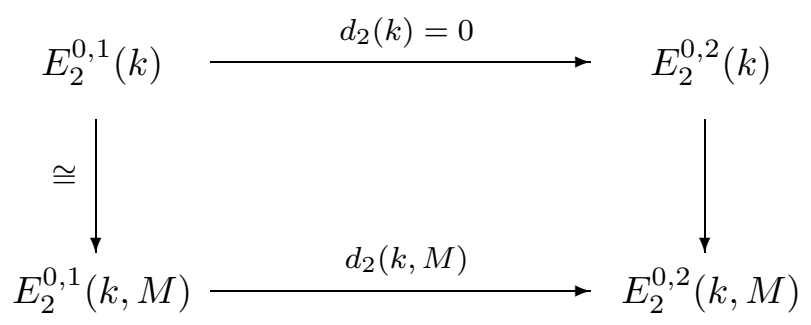

we get the vanishing of the differential $d_{2}^{0,1}(k, M)$, and, as a consequence, we get the identifications $E_{\infty}^{0,1}(k, M)=E_{2}^{0,1}(k, M) \cong E_{2}^{0,1}(k) \cong H^{1}\left(X^{k} ; \mathbb{Q}\right) / E_{2}^{1,0}(k)$.

This identifies the dimension of $H^{1}\left(p_{M+k}^{-1}(a) ; \mathbb{Q}\right)$ with the sum of dimensions of $E_{2}^{1,0}(k, M)$ and $H^{1}\left(X^{k} ; \mathbb{Q}\right) / E_{2}^{1,0}(k)$, and finally,

$$
\operatorname{dim} H^{1}\left(p_{M+k}^{-1}(a) ; \mathbb{Q}\right)=(M+k-1) \operatorname{rk} H^{1}(X) .
$$


On the other hand, there is a commutative diagram

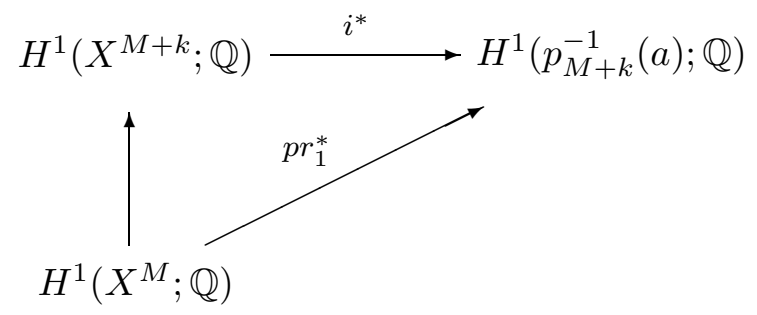

where $\mathrm{pr}_{1}^{*}$ is injective, as $E_{2}^{1,0}$ coincides with $E_{\infty}^{1,0}$ for any spectral sequence in the first quadrant, and therefore, $\operatorname{dim} H^{1}\left(X^{k} ; \mathbb{Q}\right) \geq \operatorname{dim} K e r i^{*}$. But Keri ${ }^{*}$ is a $\mathfrak{S}_{M+k}$-invariant subspace and by (2) must have dimension at least $\operatorname{rk} H^{1}(X)$. The rank of any non-trivial irreducible sub-representation of $\mathfrak{S}_{M+k}$ in $H^{1}(X)^{M+k}$ is $M+k-1$. Let $M>\left(\operatorname{dim} H^{1}(X ; \mathbb{Q})-1\right) \cdot k+1$. Then Ker $i^{*}$ is the diagonal of $H^{1}\left(X^{M+k}\right)=H^{1}(X)^{M+k}$. From the exact sequence

$$
0 \longrightarrow H^{1}(X) \stackrel{\Delta}{\longrightarrow} H^{1}\left(X^{N}\right) \longrightarrow H^{1}\left(p_{N}^{-1}(a)\right) \longrightarrow 0
$$

one gets $H^{1}\left(p_{N}^{-1}(a)\right)^{\mathfrak{S}_{N}}=0$, which is equivalent to $H^{1}\left(\hat{p}_{N}^{-1}(a)\right)=0$, when $N$ is at least $\operatorname{dim} H^{1}\left(X^{k} ; \mathbb{Q}\right)+2$.

Proof of Proposition. For large $N$ the map $p_{N}$ is surjective. Consider the map from the Albanese variety to the group of zero-cycles of degree zero modulo the equivalence, sending a point $a$ of $\operatorname{Alb} X$ to some 0 -cycle $\sum_{j=1}^{N} P_{j}-\sum_{j=1}^{N} Q_{j}=$ $\alpha^{+}-\alpha^{-}$with $p_{N}\left(\alpha^{+}\right)-p_{N}\left(\alpha^{-}\right)=a$. We have to show that this map is correctly defined and surjective.

To prove the first, one has to show that the fibers of $p_{N}$ modulo $\mathfrak{S}_{N^{-}}$-action consist of equivalent cycles, since in that case for any $\alpha^{+}, \alpha^{-} \in X^{N}$ and $\beta^{+}, \beta^{-} \in$ $X^{M}$ with $p_{N}\left(\alpha^{+}\right)-p_{N}\left(\alpha^{-}\right)=p_{M}\left(\beta^{+}\right)-p_{M}\left(\beta^{-}\right)$one has $p_{N+M}\left(\alpha^{+}, \beta^{-}\right)=$ $p_{N+M}\left(\beta^{+}, \alpha^{-}\right)$.

For an arbitrary $k$ with surjective $p_{k}$ there is a point $\gamma \in X^{k}$ such that the fiber of $\hat{p}_{N+k}$ over $p_{N+k}(\alpha \times \gamma)$ is smooth. Fix some $y \in Y_{N+k}$. There is a smooth connected projective surface in $\left(X^{N+k} \times Y_{N+k}\right) / \mathfrak{S}_{N+k}$, an appropriate complete intersection, in the fiber of $\hat{p}_{N+k}$ passing through $[\alpha \times \gamma \times y]$ and $[\beta \times \gamma \times y]$. By the Lefschetz hyperplane section theorem and Lemma 1.3, the Albanese variety is trivial.

The surjectivity is immediate from the definition.

Remark. One can easily calculate the first homology group of the homotopy fiber of the "averaged Albanese map" $\hat{p}_{N}$ as follows. The fundamental group of $(X \times Y) / \mathfrak{S}_{N}$ coincides with $\pi_{1}(X)^{N} \rtimes \mathfrak{S}_{N}$, where the symmetric group $\mathfrak{S}_{N}$ permutes the copies of $\pi_{1}(X)$ in $\pi_{1}(X)^{N}$. The fundamental group $\Gamma_{N}$ of the homotopy fiber of $\hat{p}_{N}$ is the subgroup of $\pi_{1}(X)^{N} \rtimes \mathfrak{S}_{N}$ defined by the exact 
sequence

$$
1 \longrightarrow \Gamma_{N} \longrightarrow \pi_{1}(X)^{N} \rtimes \mathfrak{S}_{N} \longrightarrow H_{1}(X) / \text { torsion } \longrightarrow 0
$$

and then

$$
\Gamma_{N}=\left\{\left(g_{1}, \ldots, g_{N} ; \sigma\right) \in \pi_{1}(X)^{N} \rtimes \mathfrak{S}_{N} \mid g_{1} \cdots g_{N} \in \Gamma_{1}\right\} .
$$

As $\left[\mathfrak{S}_{N}, \mathfrak{S}_{N}\right]=A_{N}$ is the alternating group, and for $N \geq 3$ one has the commutator identities

$$
\left[\left(g^{-1}, g, 1, \ldots\right),(12)\right] \cdot\left[\left(g^{2}, g^{-1}, g^{-1}, \ldots\right),(123)\right]=\left(g, g^{-1}, 1, \ldots\right)
$$

and $\left[\left(g, g^{-1}, 1, \ldots\right),\left(h, 1, h^{-1}, \ldots\right)\right]=([g, h], 1,1, \ldots)$, we get

$$
\left[\Gamma_{N}, \Gamma_{N}\right]=\left\{\left(g_{1}, \ldots, g_{N} ; \sigma\right) \in \pi_{1}(X)^{N} \rtimes A_{N} \mid g_{1} \cdots g_{N} \in\left[\pi_{1}(X), \pi_{1}(X)\right]\right\},
$$

and thus,

$$
H_{1}\left(\text { homotopy fiber of } \hat{p}_{N}\right)=\Gamma_{N} /\left[\Gamma_{N}, \Gamma_{N}\right] \cong H_{1}(X)_{\text {torsion }} \times \mathbb{Z} / 2 \mathbb{Z} \text {. }
$$

\section{Acknowledgements}

There was no Lemma 1.1 in the previous version of this paper. I am grateful to the referee for pointing out that the openness condition for $p_{M}$ used in the proof of Lemma 1.2 is not evident, and a statement like Lemma 1.1 is necessary.

\section{References}

[M] A. Mattuck, The irreducibility of the regular series on an algebraic variety, Illinois J. Math. 3 (1959), 145-149.

[Sa] P. Samuel, Relations d'équivalence en géométrie algébrique, 1960 Proc. Internat. Congress Math. (1958), 470-487, Cambridge Univ. Press, New York.

[Se] J.-P. Serre, Sur la topologie des variétés algébriques en caractéristique p, Symposium Internacional de Topología Algebraica, México, 1958, 24-53.

Independent University of Moscow, 121002 Moscow, B.Vlasievsky Per. 11.

Institute for Information Transmission Problems, Russian Academy of Sciences, Moscow.

E-mail address: marat@mccme.ru 